\title{
Spectral correction algorithm for multispectral CdTe x-ray detectors
}

\author{
Christensen, Erik D.; Kehres, Jan; Gu, Yun; Feidenhans'l, Robert ; Olsen, Ulrik Lund
}

Published in:

Proc. SPIE 10403, Infrared Remote Sensing and Instrumentation XXV

Link to article, DOI:

$10.1117 / 12.2272935$

Publication date:

2017

Document Version

Publisher's PDF, also known as Version of record

Link back to DTU Orbit

Citation (APA):

Christensen, E. D., Kehres, J., Gu, Y., Feidenhans'l, R., \& Olsen, U. L. (2017). Spectral correction algorithm for multispectral CdTe x-ray detectors. In Proc. SPIE 10403, Infrared Remote Sensing and Instrumentation XXV (Vol. 10391). [103930H] SPIE - International Society for Optical Engineering. Proceedings of SPIE - The International Society for Optical Engineering https://doi.org/10.1117/12.2272935

\section{General rights}

Copyright and moral rights for the publications made accessible in the public portal are retained by the authors and/or other copyright owners and it is a condition of accessing publications that users recognise and abide by the legal requirements associated with these rights.

- Users may download and print one copy of any publication from the public portal for the purpose of private study or research.

- You may not further distribute the material or use it for any profit-making activity or commercial gain

- You may freely distribute the URL identifying the publication in the public portal 


\section{Spectral correction algorithm for multispectral CdTe x-ray detectors}

\section{Erik D. Christensen, Jan Kehres, Yun Gu, Robert Feidenhans'l, Ulrik L. Olsen}

Erik D. Christensen, Jan Kehres, Yun Gu, Robert Feidenhans'I, Ulrik L. Olsen, "Spectral correction algorithm for multispectral CdTe x-ray detectors," Proc. SPIE 10393, Radiation Detectors in Medicine, Industry, and National Security XVIII, 103930H (7 September 2017); doi: 10.1117/12.2272935

Event: SPIE Optical Engineering + Applications, 2017, San Diego, California, United States 


\title{
Spectral correction algorithm for multispectral CdTe X-ray detectors
}

\author{
Erik D. Christensen ${ }^{\mathrm{a}}$, Jan Kehres ${ }^{\mathrm{b}}$, Yun $\mathrm{Gu}^{\mathrm{b}}$, Robert Feidenhans' ${ }^{\mathrm{c}}$, and Ulrik L. Olsen ${ }^{\mathrm{b}}$ \\ ${ }^{a}$ Niels Bohr Institute, University of Copenhagen, Universitetsparken 5, 2100, Copenhagen, \\ Denmark \\ ${ }^{b}$ Department of Physics, Technical University of Denmark, Fysikvej 2800, Lyngby, Denmark \\ ${ }^{\mathrm{c}}$ European XFEL GmbH,Holzkoppel 4 22869, Schenefeld, Germany
}

\begin{abstract}
Compared to the dual energy scintillator detectors widely used today, pixelated multispectral X-ray detectors show the potential to improve material identification in various radiography and tomography applications used for industrial and security purposes. However, detector effects, such as charge sharing and photon pileup, distort the measured spectra in high flux pixelated multispectral detectors. These effects significantly reduce the detectors' capabilities to be used for material identification, which requires accurate spectral measurements. We have developed a semi analytical computational algorithm for multispectral CdTe X-ray detectors which corrects the measured spectra for severe spectral distortions caused by the detector. The algorithm is developed for the Multix ME100 CdTe X-ray detector, but could potentially be adapted for any pixelated multispectral $\mathrm{CdTe}$ detector. The calibration of the algorithm is based on simple attenuation measurements of commercially avaiable materials using standard laboratory sources, making the algorithm applicable in any X-ray setup. The validation of the algorithm has been done using experimental data acquired with both standard lab equipment and synchrotron radiation. The experiments show that the algorithm is fast, reliable even at X-ray flux up to $5 \mathrm{Mph} / \mathrm{s} / \mathrm{mm}^{2}$, and greatly improves the accuracy of the measured X-ray spectra, making the algorithm very useful for both security and industrial applications where multispectral detectors are used.
\end{abstract}

Keywords: Multispectral CdTe detectors, compensation of spectral distortions, photon-counting, Multix ME100, pulse pileup correction, charge sharing correction.

\section{INTRODUCTION}

Multi-spectral CdTe detectors show great potential for improving material identification with hard X-rays ${ }^{1}$ and work in improving material identification using multi-spectral data in various radiography and tomography applications are still progressing. ${ }^{2-4}$ The Line array Multix ME100 detector is developed to perform well in high flux $\left(>1 \mathrm{Mph} / \mathrm{s} / \mathrm{mm}^{2}\right)$ applications ${ }^{5}$ and has been shown to decrease the false detection rate as compared to dual energy sandwich detectors. ${ }^{6}$ However, detector effects severely distort the measured spectrum of the Multix ME100, which could affect the detectors performance. ${ }^{7}$ Models to describe the distortion of multi-spectral detectors have been proposed previously, ${ }^{8-10}$ but algorithms for correcting the measured spectra are not fully available yet.

In this paper, we present a semi analytical computational algorithm to correct the spectrum of pixelated multi-spectral detectors, as well as show the algorithms performance on experimental data. We use the Multix ME100 v2 CdTe detector to develop and test the algorithm. The algorithm corrects the spectra for the well described effects of charge sharing, weighting potential cross talk, pulse pileup, and escape peaks, ${ }^{11,12}$ which we correct by making models based on the physical origin of these detector effects. Further, we develop a model to account for a flux dependent incomplete charge collection. The algorithm corrects each pixel's spectrum individually and is based on the method of stripping, ${ }^{13}$ correcting for each distorting effects independently. Our aim is that we with this algorithm are able to correct the measured spectra for distorting effects effectively, fast, and reliable, making the method applicable in applications like luggage screening.

Further author information:

Erik D. Christensen.: E-mail: erik.dreier@nbi.ku.dk, Telephone: +45 40638708

Radiation Detectors in Medicine, Industry, and National Security XVIII, edited by Gary P. Grim, Lars R. Furenlid, H. Bradford Barber, Proc. of SPIE Vol. 10393, 103930H · @ 2017 SPIE CCC code: $0277-786 X / 17 / \$ 18 \cdot$ doi: $10.1117 / 12.2272935$ 


\section{THE SPECTRAL DISTORTION CORRECTION MODEL}

An example of the Multix ME100's spectral response to a monochromatic beam is shown in Fig. 1. The response is influenced by a finite energy resolution and a slight shift in peak energy, another severe effect cause a low energy tail with non zero counting probability. The latter distortion arises from many factors, and using the descriptions in Ref. 11,12,14 they can be explained as follows:

- Escape peak: An X-ray photon will typically be absorbed in a semiconductor through the photoelectric effect where it excites a photo- or Auger-electron, although the latter is less likely for CdTe. For CdTe the absorption process typically produce fluorescence and the energy of the photo-electron is reduced by the energy of the released photon. For incident photons with energy above the K-shell energy, the re-emitted photon has a significant absorption length. If the K-photon is released close to the surface of the detector, it can escape the crystal and its energy will not be collected in the pixel. This creates a distortion in the acquired spectra called the escape peak phenomena.

- Charge sharing: In a pixelated detectors, the pixels are made by segmenting the anode into pads, and typically the pads are significantly smaller than the depth of the crystal to utilize the small pixel effect. ${ }^{15}$ After the photo-electron is released its energy is dissipated into the crystal through creation of electron-hole pairs. The holes and electrons drift towards the cathode and anode respectively. Much of the distortions seen in Fig. 1 arise due to cross talk between the anode pads, which occurs when the photons are absorbed close to a pixel boarder. Charge sharing then arises due to the electron charge cloud being split onto two neighboring pixels. The recorded energy is a function of the collected charge and charge sharing thus gives rise to a broad continuum of counts with energy lower than the actual photon energy.

- Weighing potential cross talk: besides charge sharing, another primary type of cross talk can occur. The weighting potential cross talk (W.P. cross talk) occurs when charges moving in the crystal above an anode induce a signal in the neighboring anode. The W.P. Cross talk is seen as an upturn in the spectrum at low energies (seen at $E<30 \mathrm{keV}$ in the figure).

- Pile up: The last primary cause to spectral distortion is the pulse pileup, which happens when two photons arrive so close in time that they are counted as one and therefore, opposite to the other effects mentioned, it is dependent on the flux density.

Other effects, such as the spectrally distortion from the difference in electron and hole mobility in $\mathrm{CdTe}^{16}$ and polarization due to charge build-up, ${ }^{17}$ are also affecting the spectrum. Both effects could cause a severe skew towards low energy of the measured spectrum at higher flux. Furthermore, the measured spectra can also show variation between pixels due to a slight miss calibration of the energy scale. In the following, all of these effects will be described in more detail and the algorithms that correct the spectrum for the distortion that they give rise to are introduced.

\subsection{Escape Peaks, Charge Sharing and Weighting Potential Cross Talk}

To perform a fast correction of the flux independent effects of charge sharing, W.P. cross talk, and the escape peak phenomena, we create an inverse detector response matrix $\mathbf{M}_{C}$ of dimension $E_{b} \times E_{b}$, where $E_{b}$ is the number of energy bins. When $\mathbf{M}_{C}$ is applied to a raw spectrum from $N$ pixels, which can be described as a matrix $\mathbf{I}_{R}$ of dimension $E_{b} \times N$, it results in the corrected spectrum for each pixel described by the matrix $\mathbf{I}_{C}$, such that

$$
\mathbf{I}_{C}=\mathbf{M}_{C} \cdot \mathbf{I}_{R} .
$$

Assuming that the response matrices are invertible and limiting the correction to the primary distortion effects, the full inverse response matrix can be calculated from

$$
\mathbf{M}_{C}=\left(\mathbf{D}_{W P} \cdot \mathbf{D}_{C} \cdot \mathbf{D}_{E}\right)^{-1},
$$




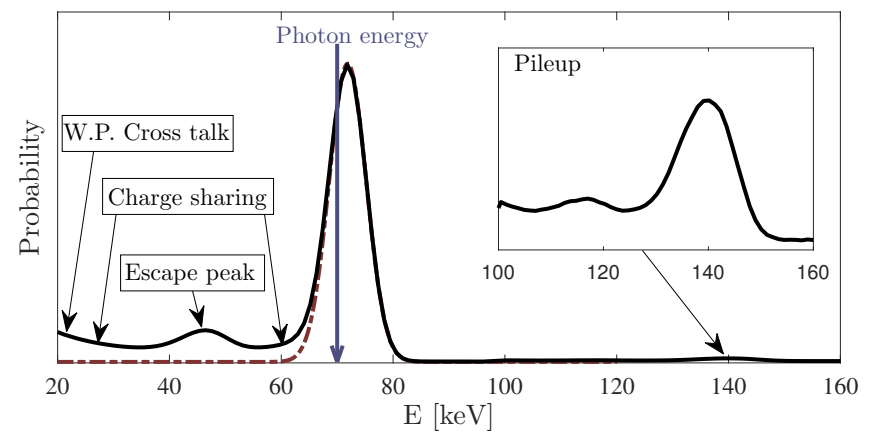

Figure 1. Spectrum measured with a Multix ME100 v2 detector at a monochromatic irradiation of $70 \mathrm{keV}$. The zoom-in on the high energy spectrum in the "Pileup box" shows the pileup peak at double energy of the primary peak. The dashed red line shows the ideal response using a finite energy resolution corresponding to the ME100 detector and illustrates the skew of the peak.

where $\mathbf{D}_{E}, \mathbf{D}_{C}$, and $\mathbf{D}_{W P}$ are the detector response matrices due to escape peaks, charge sharing, and W.P. cross talk respectively. Applying $\mathbf{M}_{C}$ according to Eqs. (1), we first correct for the W.P. cross talk, then the charge sharing, and last the escape peaks. This order was chosen as the escape peak ratio is dependent on the initial interaction of the photon, and hence should be applied to a cross talk corrected spectrum. The order of charge sharing and W.P. cross talk was chosen to remove the tail from the W.P. cross talk before correcting for charge sharing.

To create accurate response matrices, advanced models are needed and exact knowledge of the detector geometry required. It was however not the aim of our work to achieve a perfect physical model, but rather to achieve a simple post acquisition correction algorithm. For the models presented below, the following parameter are needed: The Multix ME100 detector's pixel has an area of $0.8 \times 0.8 \mathrm{~mm}^{2}$ and an active crystal thickness of $3 \mathrm{~mm}$ thickness. ${ }^{6}$ The bias voltage is assumed to be set to around $1200 \mathrm{~V} .{ }^{5}$ With these parameters, we are able to calculate the needed response matrices.

The escape peaks originate from X-ray florescence that occurs due to the X-ray photons interaction with the crystal. When the energy of the photon is larger than the $\mathrm{K}$ 1s shell binding energy, the photon will excite an electron in the ions' $\mathrm{K}$ shell through the photoelectric effect. The resulting hole will be refilled with an electron through either an Auger- or a photo-electron process. The photo-electron process results in the emission of a photon at probability given by the florescence yield. The photon is emitted in a random direction as either $K_{\alpha}$ or $K_{\beta}$ fluorescence. The energy of the fluorescence lines are for cadmium $E_{\alpha}=23.2 \mathrm{keV}$ and $E_{\beta}=26.1 \mathrm{keV}$ and tellurium $E_{\alpha}=27.5 \mathrm{keV}$ and $E_{\beta}=31.0 \mathrm{keV} .{ }^{18}$ The florescence yield is high for both $\mathrm{Cd}$ and Te ions, where 84 $\%$ and $87.5 \%$ of K-shell refilling events give rise to photon fluorescence. ${ }^{19}$ The average travel length of a K-shell photon, as calculated from the inverse of the linear attenuation coefficient of $\mathrm{CdTe},{ }^{20}$ is between $50 \mu \mathrm{m}$ and 170 $\mu \mathrm{m}$, meaning that most of the fluorescence photons are reabsorbed within the same pixel as they were emitted. However, some are absorbed in neighboring pixel and some escape the detector crystal all together. When a fluorescence photon escapes with energy $E_{i}$, the initial photons energy $E_{0}$ is recorded as $E=E_{0}-E_{i}$ by the detector.

To create the escape peak detector response matrix $\mathbf{D}_{E}$, we use that the probability $R_{i}\left(E_{0}\right)$ of having an escape photon from one of the four K-edges from Cd and Te is well described in Ref. 12 for an Amptek XR-100T CdTe pin detector. The Amptek XR-100T CdTe detector has a crystal volume to surface area fraction of 0.30 $\mathrm{mm}$. This is very close to the volume to surface area fraction of a Multix ME100's crystal of $0.31 \mathrm{~mm}$ (Each CdTe crystal is 32 pixels long and thereby $V=25.6 \cdot 0.8 \cdot 3 \mathrm{~mm}^{3}$ ), and the risk of escape peaks are therefore assumed equal. The risk of florescence photons escaping the pixel and being absorbed in the neighboring pixels is assumed negligible compared to the other cross talk effects. Taking only the photons that escapes the entire crystal into account, the normalized counting probability $D_{E}(E)$ of counting a photon with energy $E$ in the 
detector due to an incoming photon with energy $E_{0}$ is given by

$$
\begin{gathered}
D_{E}\left(E_{0}\right)=\sum_{i}^{4}\left(1-R_{i}\left(E_{0}\right)\right) \\
D_{E}\left(E_{0}-E_{i}\right)=R_{i}\left(E_{0}\right) .
\end{gathered}
$$

To create the response matrices for charge sharing, $\mathbf{D}_{C}$, and W.P. cross talk, $\mathbf{D}_{W P}$, we use a single photon Monte Carlo simulation of a Multix ME100 pixel's response to an X-ray beam. The simulation is done at an energy interval corresponding to the energy bin interval of the Multix ME100. In the simulation, a pixel is homogeneously illuminated by photons across the surface. The probability of photon absorption as function of depth in the crystal was simulation according to the expected attenuation of CdTe. ${ }^{20} \mathbf{D}_{C}$ and $\mathbf{D}_{W P}$ are found from the simulation's distribution of photons as function of recorded and incoming photon energy. Below, the energy recorded by the detector as function of energy and position of a single photon is described for charge sharing and W.P. cross talk.

In the simulation, the amount of charge shared between two pixels for each photon is calculated by assuming an 1D Gaussian charge distribution parallel to the Multix ME100 pixel array. In this model, the energy recorded in the neighboring pixels to the "left" or "right" of the pixel where the photon of energy $E_{0}$ was absorbed, is given by ${ }^{21}$

$$
\begin{aligned}
E_{\text {right }} & =\frac{E_{0}}{2}\left(1-\operatorname{erf}\left(\frac{d_{x} / 2-x}{\sigma \sqrt{2}}\right)\right) \\
E_{\text {left }} & =\frac{E_{0}}{2}\left(1-\operatorname{erf}\left(\frac{d_{x} / 2+x}{\sigma \sqrt{2}}\right)\right),
\end{aligned}
$$

where $d_{x}$ is the width of the pixel and $\sigma$ is the standard deviation of the charge cloud distribution. The energy recorded in the center pixel is given by $E_{\text {center }}=E_{0}-E_{\text {right }}-E_{\text {left }}$

Two properties determine the charge cloud size $\sigma_{t}$; the charge diffusion and the charge repulsion perpendicular to the electric field. Combined with the initial width of the excited charge cloud $\sigma_{i}$, the total width of the charge cloud at the segmented anode is assumed given by

$$
\sigma=\sqrt{\sigma_{t}^{2}+\sigma_{i}^{2}}
$$

$\sigma_{t}$ can be calculated from the diffusion equation

$$
\sigma_{t}^{2}=2 D \tau
$$

where $D$ is the diffusion constant and $\tau$ is the charge carrier lifetime. ${ }^{14}$ To include the charge repulsion in this model, an effective diffusion constant is used ${ }^{22}$

$$
D_{\text {eff }}=D+\frac{1}{15}\left(\frac{3 \mu_{e} N q}{4 \pi \epsilon}\right) \frac{1}{\sqrt{5} \sigma_{i}} .
$$

Above, $\mu_{e}$ is the electron mobility and $q$ is the elementary charge. The number of charges per photon is given by $N=E_{e} / \Delta_{E}$, where $\Delta_{E}=4.43 \mathrm{ev} / \mathrm{ehp}^{23}$ is the energy per electron-hole pair (ehp) for CdTe, and $E_{e}$ is the energy of the excited photo-electron. To account for the K-edge excitation, the photo-electron's energy is set equal to $E_{e}=E_{p h}-26 \mathrm{keV}$ assuming that the fluorescence photon is not contributing to the charge cloud size. The additional contribution to the charge cloud distribution due to reabsorbed fluorescence photon creating two cloud centers is thereby not taken into account. This choice was made to simplify calculations of the charge sharing effect.

By combining Eqs. (6) and (7), we obtain an expression for the cloud width due to repulsion and diffusion

$$
\sigma_{t}=\sqrt{2 \tau D+\frac{2 \tau}{15}\left(\frac{3 \mu_{e} N q}{4 \pi \epsilon}\right) \frac{1}{\sqrt{5} \sigma_{i}}} .
$$


According to the Einstein relation $D=\frac{\mu_{e} k_{B} T}{q}$, where $T$ is the temperature and $k_{B}$ is the Boltzmann's constant. Assuming parallel plate electrodes with bias voltage $U$, we can rewrite the drift time in terms of detector depth $d$ and interaction point compared to the anode $z$, such that $\mu_{e} \tau=\frac{z d}{U} \cdot{ }^{24}$ By combining the above with Eqs. (8) and (5) we find

$$
\sigma_{t}=\sqrt{2 \frac{k_{B} T z d}{q U}+\left(\frac{z d N q}{10 \pi \epsilon U}\right) \frac{1}{\sqrt{5} \sigma_{i}}+\sigma_{i}^{2}} .
$$

The W.P. cross talk phenomenon originates from how the charge is collected in the pixel's anode. The electrical signal in a semiconductor detector's electrodes arises due to the movement of the charges in the crystal. The induced charge $Q$ on an electrode due to moving charges in the active detector volume is described by the Shockley-Ramo theorem ${ }^{14,25,26}$

$$
Q=N_{0} \cdot q \cdot \Delta \phi_{0},
$$

where $N_{0}$ is the number of charge carrier, $q$ is the charge of the carriers, and $\Delta \phi_{0}$ is the weighting potential difference from start to end of the charge path. An anode's weighing potential extends beyond the pixel's limits, as a result charges moving in the neighboring pixel can create a small signal in the pixel itself. The W.P. cross talk is in our simulation modeled by rewriting Eqs. (10) into

$$
\Delta E=E_{0} \cdot \Delta \phi_{0}(r),
$$

where $\Delta E$ is the energy deposited in pixel $j$ and $E_{0}$ is the energy of the photon absorbed in pixel $j-1 . \Delta \phi_{0}$ is now the difference in weighting potential of pixel $j$ from start to end of the charge path.

A simple model of the weighting potential $\phi_{0}(r)$ is described in Refs. 11, where it is assumed that the detector consists of two infinite parallel plates. This model is taken as a simple approximation of the W.P. cross talk in the Multix ME100, and it is repeated here for the ease of the reader. Assuming a rectangular anode pad, the $j$ pixel's weighting potential at any interaction point above pixel $j-1$, is given by the following relation

$$
\begin{aligned}
\phi_{0}(x, y, z)=\frac{1}{2 \pi} & {\left[\arctan \left(\frac{(a-\xi)(b-\eta)}{z \sqrt{(a-\xi)^{2}+(b-\eta)^{2}+z^{2}}}\right)+\arctan \left(\frac{(a-\xi) \eta}{z \sqrt{(a-\xi)^{2}+\eta^{2}+z^{2}}}\right)+\ldots\right.} \\
& \left.\arctan \left(\frac{\xi(a-\xi)}{z \sqrt{\left(\xi^{2}+(b-\eta)^{2}+z^{2}\right.}}\right)+\arctan \left(\frac{\xi \eta}{z \sqrt{\xi^{2}+\eta^{2}+z^{2}}}\right)\right]
\end{aligned}
$$

where

$$
\begin{aligned}
& \xi=\frac{\left(x-x_{1}\right)\left(x_{2}-x_{1}\right)+\left(y-y_{1}\right)\left(y_{2}-y_{1}\right)}{a} \\
& \eta=\frac{-\left(x-x_{1}\right)\left(y_{2}-y_{1}\right)+\left(y-y_{1}\right)\left(x_{2}-x_{1}\right)}{b},
\end{aligned}
$$

here $a$ and $b$ are the dimensions of the anode pad, with the corners of the anode being placed at $\left(x_{i}, y_{i}\right)$ for $i=1 . .4$ and $x, y, z$, is the simulated interaction point of the charge above pixel $j-1$ with respect to the center of anode $j$. How $a$ and $b$ were determined is described in the laboratory experiments sections.

The combined response and inverse response matrices from the charge sharing, W.P. cross talk and escape peaks are shown in Fig. 2.

\subsection{Pulse Pileup}

Pulse pileup occurs due to the overlapping of electrical pulses generated in an anode by two photons arriving close in time in the same pixel. To correct for the effect of pulse pileup, we use the iterative approach described by Plagnard. ${ }^{12}$ The approach is based on the assumption that two photons with energy $E_{1}$ and $E_{2}$ will be counted as one with the combined energy of the two $E_{t}=E_{1}+E_{2}$, if the time between the two is small enough. The 


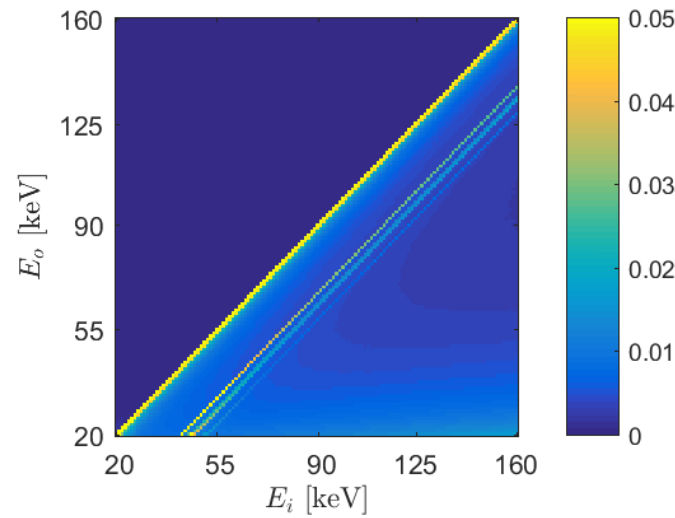

(a)

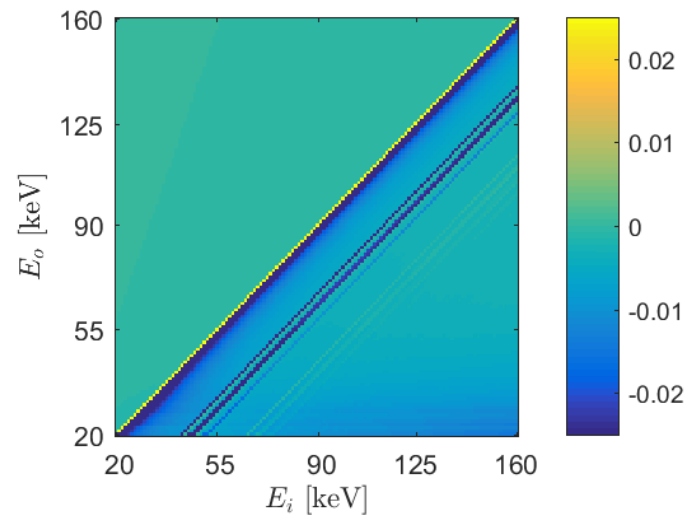

(b)

Figure 2. The simulated detector response matrix $\mathbf{D}_{W P} \cdot \mathbf{D}_{C} \cdot \mathbf{D}_{E}$ (a) and inverse detector response matrix $\left(\mathbf{D}_{W P} \cdot \mathbf{D}_{C} \cdot\right.$ $\left.\mathbf{D}_{E}\right)^{-1}(\mathrm{~b})$.

affect of the pulse pile up on the measured spectrum $I_{R}(E)$ can be described by the pileup spectrum $I_{P U}\left(E_{n x}\right)$. The pileup correction algorithm needs to calculate $I_{P U}\left(E_{n x}\right)$ for all values of $E_{n}$ and $E_{x}$, with $E_{n}$ and $E_{x}$ being the center energy of the Multix ME100's $n$ and $x$ energy bin respectively. The pileup effect shifts the spectrum from low to high energy, and hence the algorithm needs to step from lowest $n$ value to highest. For each $n$, the pileup spectrum is calculated for the hole range of $x$ by using

$$
I_{P U}\left(E_{n x}\right)=\frac{I_{R}\left(E_{n}\right)}{\sum_{e} \bar{I}_{R}\left(E_{e}\right)} \cdot C_{P U} \cdot \bar{I}_{R}\left(E_{x}\right) .
$$

Afterwards, the obtained spectrum $I_{P U}(E)$ is subtracted from the raw spectrum and the summed contribution is added to the spectrum value $I_{R}\left(E_{n}\right)$ of the $n$ energy bin to obtain the corrected spectrum

$$
\begin{aligned}
I_{C}(E) & =I_{R}(E)-I_{P U}(E) \\
I_{C}\left(E_{n}\right) & =I_{R}\left(E_{n}\right)+\sum_{e} I_{P U}\left(E_{e}\right) .
\end{aligned}
$$

At this point $n$ is increased and the procedure is repeated. The coefficient $C_{P U}$ in Eqs. (14) is in Plagnard determined by the operator, but we instead propose an automatic fitting approach using the attenuation curve of aluminium. This approach is described below in the laboratory experiments section.

\subsection{Slow Hole Mobility and Polarization}

CdTe suffers from a large difference in the drift mobility of the electrons, $\mu_{e}=1000 \mathrm{~cm}^{2} / \mathrm{V}$, and holes, $\mu_{h}=80$ $\mathrm{cm}^{2} / \mathrm{V} .{ }^{23}$ It is well known that the large difference will cause an incomplete charge collection which results in some photons being registered with lower energy than their actual, thereby causing a skew of the measured spectrum towards lower energy. ${ }^{16}$ Furthermore, effects like polarization from the build up of charge over time will push the measured spectrum towards lower energy as well. ${ }^{17}$ It is our assumption that both of these two effects will increase with flux. To correct for this problem, we suggest a simple empirical algorithm that restores the spectrum to the actual photon energy spectrum. The approach will be referred to as the SDC (peak Skew Distortion Correction) algorithm. The approach is iterative, starting from the highest energy bin of the spectrum $I(E)$ and moving down. For each bin $n$, the SDC algorithm subtracts a function $G(E)$ from the spectrum below the present energy $E_{n}$, to account for the smearing of the spectrum towards lower energy. The summed $G(E)$ is then added to the spectrum at present energy bin $n$. The method can for each step $n$ be summarized as 


$$
\begin{aligned}
G(E) & = \begin{cases}\frac{C_{S D C} I\left(E_{n}\right)}{\sum_{e} I\left(E_{e}\right)} \exp \left(\frac{\left(E-E_{n}\right)^{2}}{2 \sigma^{2}}\right) & \text { if } E \leq E_{n} \\
0 & \text { if } E>E_{n}\end{cases} \\
I(E) & =I(E)-G(E) \\
I\left(E_{n}\right) & =I\left(E_{n}\right)+\sum_{e} G\left(E_{e}\right) .
\end{aligned}
$$

Following this, $n$ is decreased by one bin and the procedure is repeated. The function $G(E)$ has two scalable parameters; the assumed flux independent width $\sigma$ and the flux scaling constant $C_{S D C}$. We introduce how these were determined in the experimental sections.

\subsection{Energy Calibration}

Fluctuation in the measured energy on the order of a few keV can occur between the pixels in the Multix ME100 spectrum. To calibrate the detector for these variations, the spectrum of the radioactive isotope ${ }^{57}$ Co is measured with the Multix ME100. For each pixel's measured spectrum, the peak center of the primary and secondary radiation lines of the isotope as well as the escape peaks can be found. By fitting a 1st degree polynomial to the expected peak centers as function of the measured peak centers, a conversion from the measured to actual energy is found.

All correction methods have now been introduced, and we have thereby arrived at our final correction approach: First, each pixel's energy scale is recalibrated according to the above. Next, the spectrum is corrected for the flux independent phenomena like charge sharing, WP. cross talk, and escape peaks according to Eqs. (1). Finally, the pileup is corrected according to Eqs. (15), followed by the SDC algorithm in Eqs. (16).

\section{SYNCHROTRON EXPERIMENTS}

Synchrotron experiments were conducted at the materials science beamline ID11, at the European Synchrotron Radiation Facility (ESRF). The aim of these experiments were to evaluate the energy and position dependence of the different distorting effects. At the ID11 beamline, the Multix ME100 detector's response to monochromatic $\mathrm{X}$-ray radiation was measured at 8 different energies between 24 and $138 \mathrm{keV}$. The X-ray energy selection was provided by double bent crystal monochromator operating in horizontal focusing Laue geometry. Further, the detector response as function of X-ray flux was also measured. The Multix detector was mounted on the camera stage in the EH3 hutch allowing for movement in the plane perpendicular to the beam. The beam size onto the detector was controlled using slits placed just in front of the EH3 sample stage. To obtain a reference measurement to validate the energy of the monochromatic beam, a high energy resolution Amptek XR100T $\mathrm{CdTe}$ pin detector was mounted on the sample stage. The monochromatic X-ray beam's energy distribution FWHM was, using the Amptek detector, determined to be below $1.4 \mathrm{keV}$ (this is at the Amptek resolution limit and the beam's energy distribution FWHM is thought to be much smaller). An X-ray beam energy distribution FWHM of $1.4 \mathrm{keV}$ is well below the energy resolution of the Multix detector.

\subsection{Evaluating the Charge Sharing Algorithm}

Controlling the beam size with the slits in front of the sample and the movement of the detector relative to the beam with the detector stage, the Multix ME100's response to a monochromatic X-ray beam slitted down to $5 \times 5 \mu \mathrm{m}^{2}$ was measured. The beam was moved parallel and perpendicular to the pixel array in steps of $\sim 20$ $\mu \mathrm{m}$. The scan parallel to the pixel array was made across two pixels starting from approximately the center of a pixel as shown in Fig. 3a. The detector response was measured for $2 \mathrm{~s}$ at each beam position before moving the detector.

In Fig. 3b, the result of a scan at $E=120 \mathrm{keV}$ can be seen as function of beam position and measured energy of the beam. The figure shows how charge is shared between pixels around the pixel borders. By summing the total intensity at each pixel, we find that the cross talk extends around $200 \mu \mathrm{m}$ into the neighboring pixel, as shown in figure 4a. Fitting the intensity around the border between two pixels with a combination of a broad 


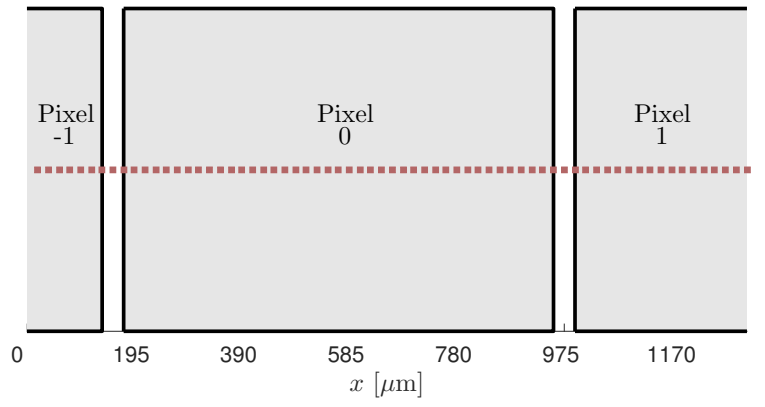

(a)

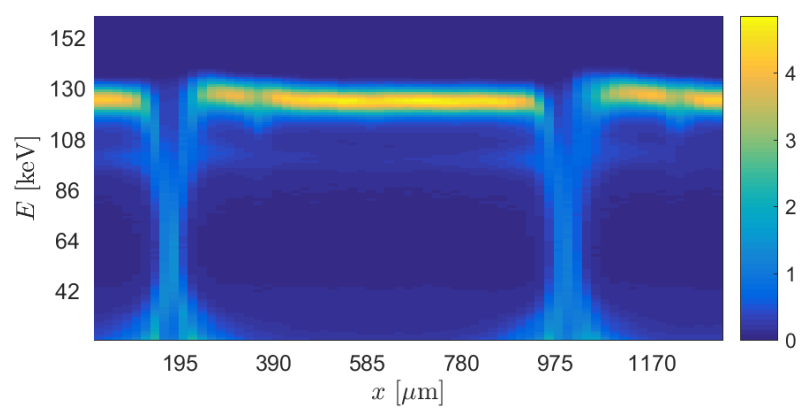

(b)

Figure 3. The experimental procedure for the parallel scan along the Multix ME100 pixels (a). The resulting detector response as function of readout energy and the spatial position of the monochromatic pencil beam at an incoming $\mathrm{x}$-ray energy of $120 \mathrm{keV}$ (the color scale is in $\mathrm{kph} / \mathrm{s}$ ) (b).

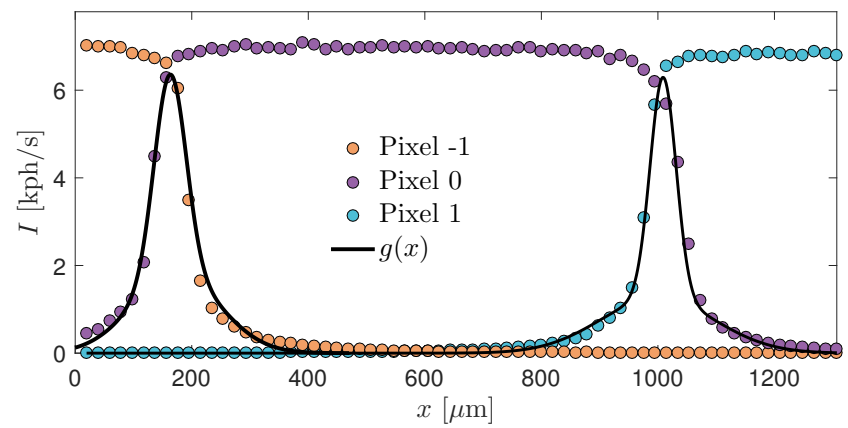

(a)

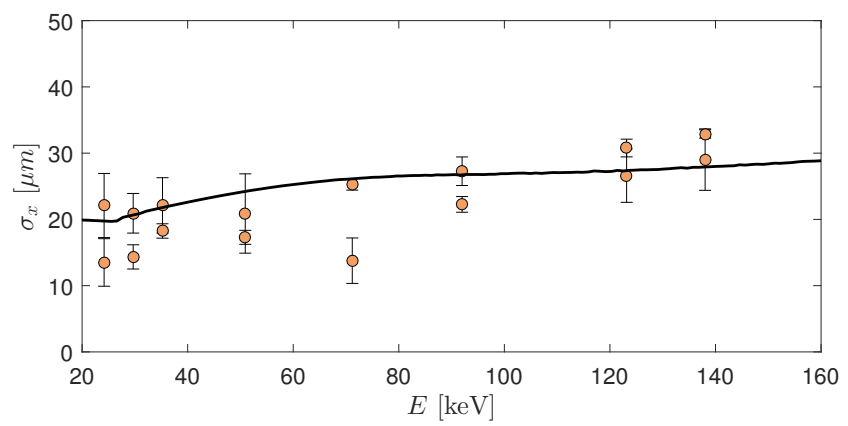

(b)

Figure 4. The summed intensity of each pixel as function of spatial position of the monochromatic X-ray beam fitted with double Gaussian function $g(x)$ at both pixel borders (a) (The shown data is the same as the data presented in Fig. 3b). The width of the narrow peak of $g(x)$ plotted as function of the incoming monochromatic beam energy (colored circles) with the estimated electron cloud width (black line), given by Eqs. (9), superimposed (b).

and a narrow Gaussian distribution $g(x)$, we can obtain an estimate of the charge sharing distribution. The means of the two Gaussian functions are forced equal. It is assumed that the charge sharing is governed by the cloud size, and that the cloud size is described by the narrow Gaussian function of $g(x)$. To evaluate the model in Eqs. (8), we make similar fits for all 8 energies for both of the two pixel boarders reached in the parallel scan. In Fig. 4b, the width of the narrow Gaussian distribution is shown for each energy with the model in Eqs. (9) superimposed on the data. Since the model seems to describe the width of the narrow Gaussian distribution at each energy well, we take Eqs. (9) as a good description of the charge cloud width.

The broad Gaussian distribution of $g(x)$ is primarily used for fitting purposes. The origin of this broader and weaker charing of charge between pixels could be the weighting potential cross talk, or could even originate from the K-florescence photons escaping into the neighboring pixel. The width of the broad Gaussian distribution is of the order of $100 \mu m$, fitting well with escape photons mean travel length between $50 \mu m$ and $170 \mu m$. This phenomenon was not studied further.

\subsection{Finding the Parameters for the SDC Algorithm}

The $\sigma$ parameter of the SDC algorithm described Eq. (16) was determined from flux dependence scans at ID11. The flux scans were made by increasing the beam opening in steps from $5 \times 5 \mu \mathrm{m}^{2}$ to $65 \times 65 \mu \mathrm{m}^{2}$ with a beam positioned at the center of a pixel. From the position scans, it was estimated that the cross talk between pixels did not extend far enough into the the pixel to affect this measurement. In Fig. 5a, the result of such a scan is shown for a $E=50 \mathrm{keV}$ monochromatic beam. In the figure, the escape peak around $E=25 \mathrm{keV}$ and the pulse pileup peak at $E=100 \mathrm{keV}$ are clearly seen. Further, the spectrum shows a clear distortion towards lower energies as the beam flux is increased. 


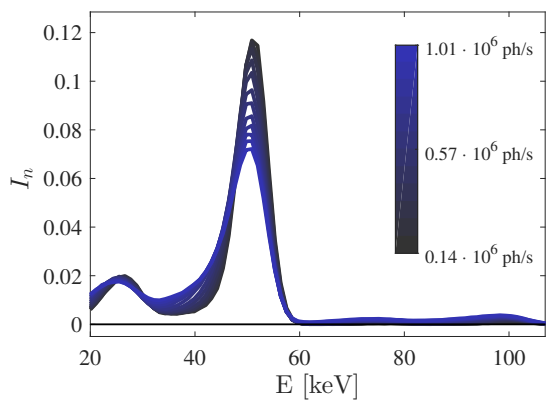

(a)

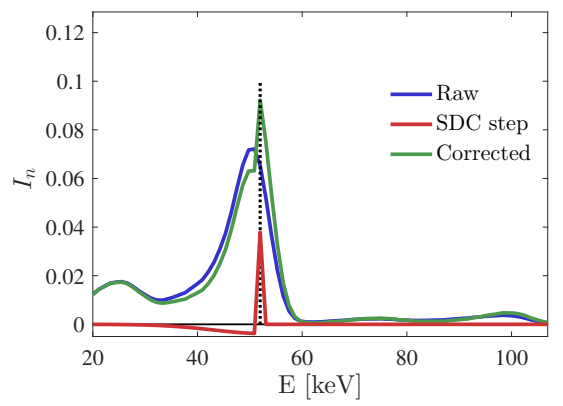

(b)

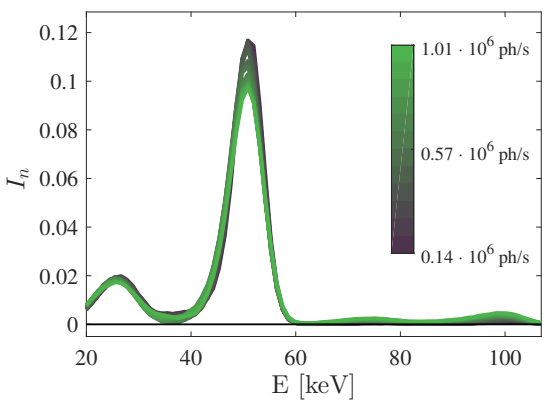

(c)

Figure 5. Applying the SDC algorithm to a spectrum. The raw spectra acquired at an incoming monochromatic X-ray beam of $E=50 \pm 0.3 \mathrm{keV}$ at different intensities. The X-ray intensity is given in the legend in units of count rate of the Multix ME100 detector (a). The raw spectrum $I(E)$ (Raw) is iteratively corrected from high to low energy by adding a correction spectrum (SDC step), described by the SDC algorithm in Eqs. (16), resulting in the corrected spectrum (Corrected) (b). The $E=50 \pm 0.3 \mathrm{keV}$ spectra corrected with the SDC algorithm (c).

By applying the SDC algorithm, the distortion of the spectrum seen in Fig. 5a can be mitigated. By adjusting the $\sigma$ parameter, the correction of similar spectra obtained at other energies can be optimized. The best value of the parameter of the SDC algorithm was found to be at $\sigma=10 \mathrm{keV}$. Fig. 5b shows how the SDC algorithm is applied to a measured spectrum. The figure illustrates how the SDC algorithm shifts the spectrum towards higher energies at each step. The algorithm works well at correcting the spectra for the peak skew as function of flux, as can be seen when comparing Fig. 5a and Fig. 5c.

\section{LABORATORY EXPERIMENTS}

The correction algorithm was evaluated and optimized through several experiments at a laboratory X-ray instrument at the Technical University of Denmark. ${ }^{27}$ All laboratory experiments used a COMET MXR-160HP/11 tube with a tungsten (W) target and a COMET MXR generator capable of running up to a voltage of $160 \mathrm{kVp}$ and current of $22.5 \mathrm{~mA}$. By using tungsten slits, we were able to collimate the beam and reduce background effects. The spectra were generated by changing the source voltage and inserting different attenuation filters in between the source and the detector.

\subsection{Testing the Flux Independent Algorithms}

To evaluate the performance of the correction algorithm in correcting the flux independent effects; charge sharing, W.P. cross talk, and escape peaks, an experiment was performed where the source current was kept to a minimum to reduce flux on the detector. This was useful to determine the best value for the parameter $a$ and $b$ in Eqs. (13). In theory $a$ and $b$ should be equal to the size of the anode pad. However, the W.P. cross talk model is an approximation, and the anode pad size is therefore only seen as a scaling parameter rather than an actual physical constant. To validate the experiments, the same spectrum was measured with the Amptek XR100T CdTe pin detector designed to have a significantly better energy resolution than the Multix ME100 in a low flux experiment. The Amptek spectrum was used as a reference spectrum assumed to describe the actual spectrum. The best correction of the Multix data was found with $a=b=0.65 \mathrm{~mm}$. It was found that the correction algorithm underestimates the effect of W.P. cross talk if the measured X-ray spectrum is affected by severe beam hardening from high $Z$-filters, like copper. This effect is mitigated by increasing the W.P. correction by a factor of $1+E_{p} /(160 \mathrm{keV})$ if $E_{p}>100 \mathrm{keV}$, where $E_{p}$ is the energy where spectrum has its maximum.

Two corrected spectra are shown in Fig. 6. To compare the corrected Multix and Amptek spectrum, the Amptek spectrum is convoluted with a Gaussian kernel of $\sigma=4 \mathrm{keV}$ corresponding to the lower energy resolution of the ME100 detector. As it can be seen from the figure, the correction works decent compared to the reference measurement. Qualitatively, this was the case for all spectra we tested. 


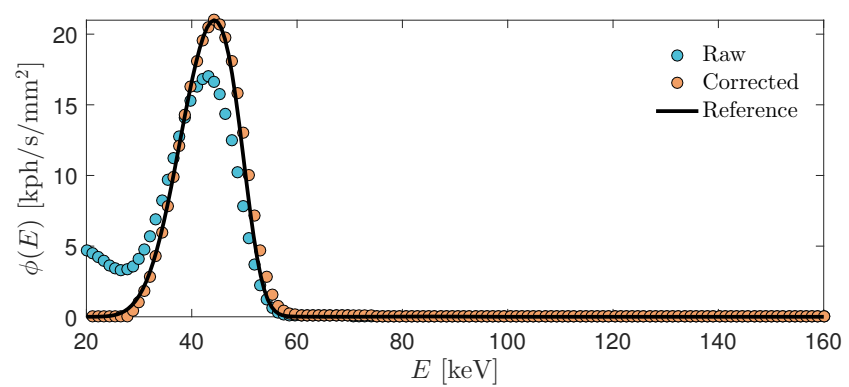

(a)

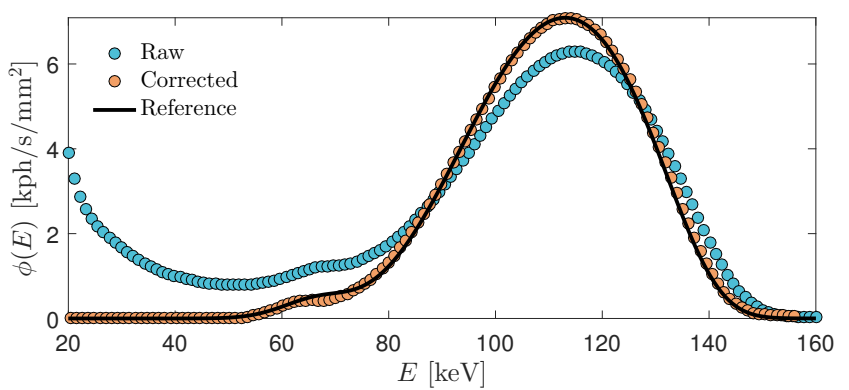

(b)

Figure 6. Comparison between Multix spectra corrected with the correction algorithm (Corrected), raw spectra (Raw), and reference spectra obtained with Amptek XR100 (Reference). The reference spectra are scaled to fit the corrected data. The spectra were obtained using a filter of $19.9 \mathrm{~mm}$ aluminium at a source voltage of $U=50 \mathrm{kV}$ (a) and $5.96 \mathrm{~mm}$ copper and $0.2 \mathrm{~mm}$ tantalum at $U=140 \mathrm{kV}(\mathrm{b})$.

\subsection{Optimizing the Flux Dependent Coefficients}

The proposed flux dependent coefficients $C_{P U}$ and $C_{S D C}$, presented in Eqs. (14) and (16) respectively, need to be identified from experiments. To do this, we used a measurement of the linear attenuation coefficient of different thickness of aluminium and for different flat field fluxes. The linear attenuation is calculated from the measurements as follows:

$$
\mu(E)=-\frac{1}{x} \log \left(\frac{I(E)}{I_{0}(E)}\right)
$$

where $I(E)$ is the measured X-ray spectrum of the X-ray beam that has passed through the material, $I_{0}(E)$ is the flat field spectrum, and $x$ is the thickness of the material. Since the linear attenuation coefficient is a material constant independent of thickness, it can be used as a reference measure of how well the correction algorithm performs. The spectral distortion affects both the spectrum of the beam that has passed through the material and the flat field spectrum, and therefore the the linear attenuation coefficient is largely affected by the distortion of the measured spectra.

The first measurement of the linear attenuation was done by placing aluminium plates of 6 different thickness between the detector and source. The experiment was repeated for 4 different flat field fluxes. An example of an experiment at flat field flux of $3.7 \mathrm{Mph} / \mathrm{s} / \mathrm{mm}^{2}$ is seen in Fig. 7. As it can be seen from the figure, the spectrum is highly distorted due to the different effects described above. A clear drop in attenuation coefficient is seen around $E=59 \mathrm{keV}$ and a smaller drop around $E=65 \mathrm{keV}$. The drops in attenuation coincide with the tungsten $K_{\alpha}$ and $K_{\beta}$ peaks $\left(E_{\alpha}=59.3 \mathrm{keV}\right.$ and $E_{\beta}=67.2 \mathrm{keV}$ respectively $\left.{ }^{28}\right)$ from our source, and we therefore expect that the drop in attenuation is due to a distortion of the tungsten peaks from the flux dependent skew that was observed in figure 5 .

By adjusting $C_{P U}$ and $C_{S D C}$ for both the $I(E)$ and the $I_{0}(E)$ spectrum, we obtain a best fit between any measured attenuation curve of aluminium and the theoretical expected, ${ }^{20}$ using the $\chi^{2}$ as measure of the quality of the fit. The measured linear attenuation is individually fitted to the theoretical for the $n$ different thicknesses of aluminium for each flat field flux. By doing so, $n C_{P U}$ and $C_{S D C}$ values are obtained for both the flat field and attenuated spectra. Afterwards, the $n$ flat field correction coefficients $C_{P U}$ and $C_{S D C}$ are averaged. The linear attenuation fitting is then repeated, but this time using the average $C_{P U}$ and $C_{S D C}$ to correct the flat field spectrum as an initial guess. Repeating this procedure ensures that the flat field spectrum correction is similar for all thicknesses.

In Fig. 8, the $C_{P U}$ and $C_{S D C}$ values that result in the best fit between measured and theoretical linear attenuation coefficient are shown for 21 spectra. These 21 spectra correspond to 3 sets of measurement, each containing a flat field spectrum $I_{0}(E)$ and 6 attenuated spectra $I(E)$. The coefficient of the sets are found independently of each other. The $C_{P U}$ and $C_{S D C}$ coefficients are fitted with a 1 st order polynomial. The polynomials are used to generate fast lookup tables for correcting the spectra. Negative fit function values are set equal to zero in the plot as well as the lookup table. 


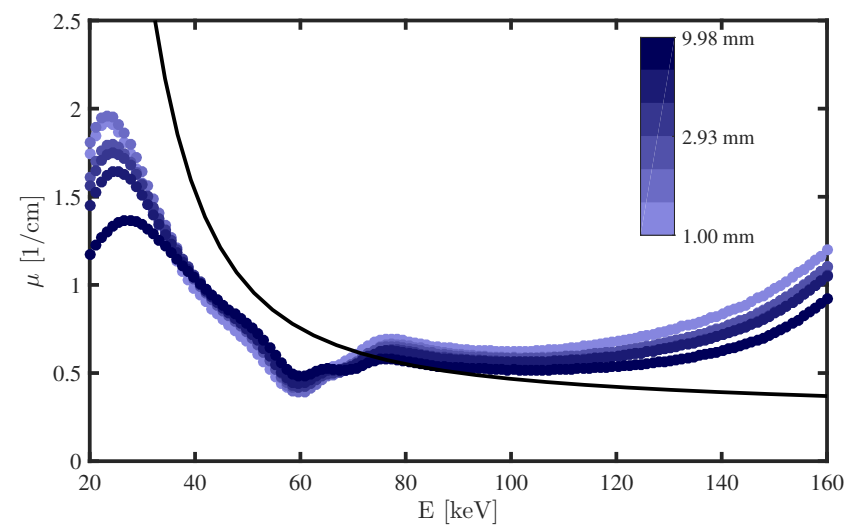

Figure 7. The attenuation of aluminium from uncorrected measurements. Different thicknesses of aluminium was used as given in the color scale. The black curve shows the theoretical expect linear attenuation. ${ }^{20}$ The flat field flux of all measurements was $3.7 \mathrm{Mph} / \mathrm{s} / \mathrm{mm}^{2}$.

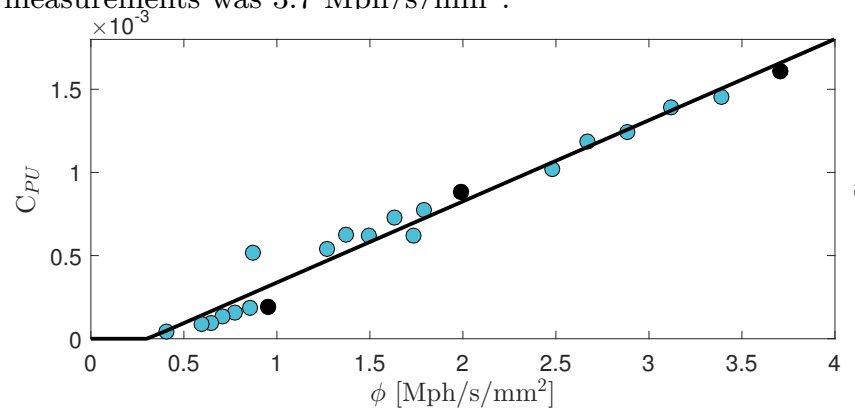

(a)

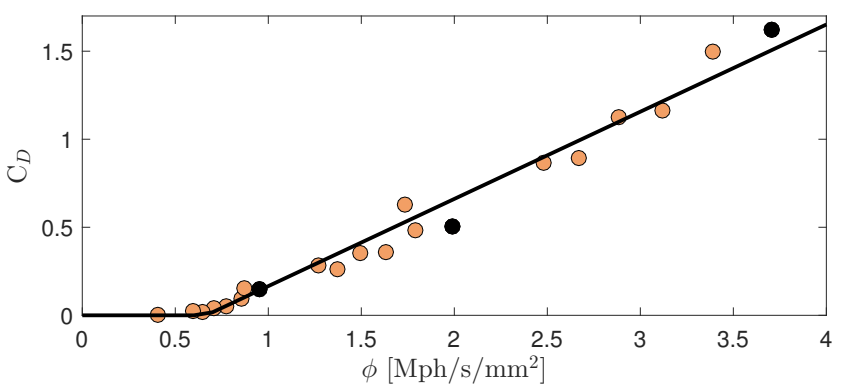

(b)

Figure 8. Pileup, $C_{P U}$, (a) and SDC, $C_{S D C}$, (b) coefficient found from fitting the linear attenuation measured with the Multix ME100 to the theoretical linear attenuation curve of aluminium. Each point represents a corrected spectrum. The colored circles represent the correction coefficient used for the $3 \times 6$ attenuated spectra from the 6 different thicknesses of aluminium at 3 different flat field fluxes. The black circles represent the coefficients used for the 3 flat field spectra. The black lines are the $C_{P U}$ and $C_{S D C}$ lookup tables, explained in the main text.

\section{FINAL VALIDATION}

To test the final correction algorithm, we used measurements of the linear attenuation coefficient of PVC, aluminium, copper, and tantalum. As done for the calibration measurements described above, the attenuation was measured at different fluxes. By applying the calibration algorithm to the flat field and attenuated spectra and calculating the linear attenuation coefficient from these, we obtain the corrected linear attenuation curve. The measured and the corrected linear attenuation curves can be seen for a flat field flux $\Phi_{0}=4.7 \mathrm{Mph} / \mathrm{s} / \mathrm{mm}^{2}$ in Fig. 9. In the figure, the corrected curves do not extend to $20 \mathrm{keV}$ for all materials. This is due to the attenuation coefficient diverging towards infinite when the corrected energy bins $I_{C}(E)$ contains close to zero photons and the signal to noise ratio converges to infinite values. The divergence can in particular be seen on the corrected attenuation curves of the heavier elements, copper and tantalum, where the beam is attenuated so heavily that no photons below a certain energy reach the detector. The same effect was seen for thicker sheets of aluminium and PVC at low flux. From Fig. 9, it can be seen that the corrected attenuation curve match the theoretical expected much better. At low energy $E<50 \mathrm{keV}$, the correction of charge sharing and W.P. cross talk clearly gives a much better fit between theory and data. Furthermore, the results obtained for all the materials show that the algorithm is consistent across a large range of attenuation coefficients.

The performance of the correction algorithm is quantified by calculating the correlation coefficient between the different curves. In Tab. 1, we show the average correlation coefficient between $N$ sheets of different thickness. The average correlation coefficient is given by $\left\langle\rho_{1, j}\right\rangle_{C}=\frac{1}{N} \sum_{j}^{N} \rho_{1, j}$, where $\rho_{1, j}$ is the correlation coefficient between the thinnest of the measured sheets, 1 , and the $j$ 'th measurement. Similarly, the table also shows the average correlation between theory $\left\langle\rho_{t, j}\right\rangle_{C}=\frac{1}{N} \sum_{j}^{N} \rho_{1, j}$ and the $j$ 'th thickest sheet. As can be seen from the 


\begin{tabular}{|c||c|c||c|c|}
\hline \multicolumn{1}{|c||}{ Material } & \multicolumn{2}{c||}{ Self correlation } & \multicolumn{2}{c|}{ Correlation with theory } \\
\hline & $\left\langle\rho_{1, j}\right\rangle_{R}$ & $\left\langle\rho_{1, j}\right\rangle_{C}$ & $\left\langle\rho_{t, j}\right\rangle_{R}$ & $\left\langle\rho_{t, j}\right\rangle_{C}$ \\
\hline PVC & 0.874 & 0.989 & 0.745 & 0.982 \\
\hline Aluminium & 0.966 & 0.996 & 0.671 & 0.849 \\
\hline Copper & 0.919 & 0.994 & 0.962 & 0.997 \\
\hline Tantalum & 0.674 & 0.937 & 0.733 & 0.946 \\
\hline
\end{tabular}

Table 1. The average self correlation coefficient between measured attenuation curves from different thicknesses of material and the average correlation coefficient between the curves and the theoretical expected. Both calculated as explained in the main text. $R$ is the raw data and $C$ is the corrected.

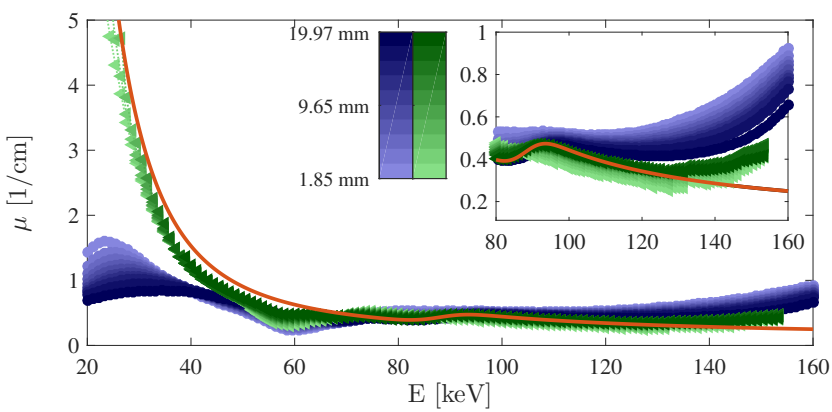

(a)

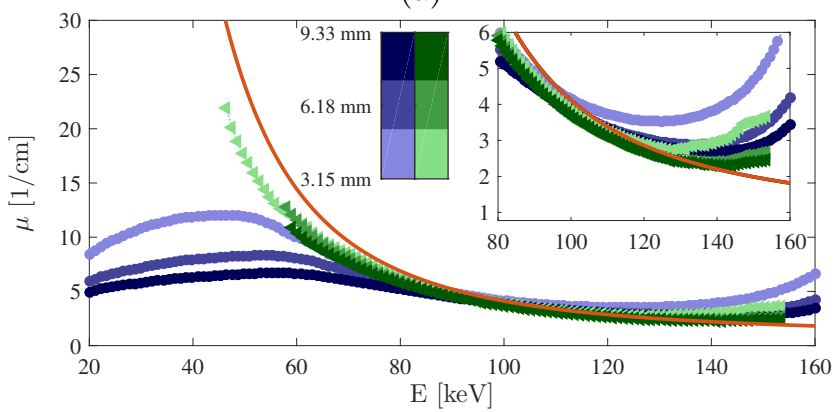

(c)

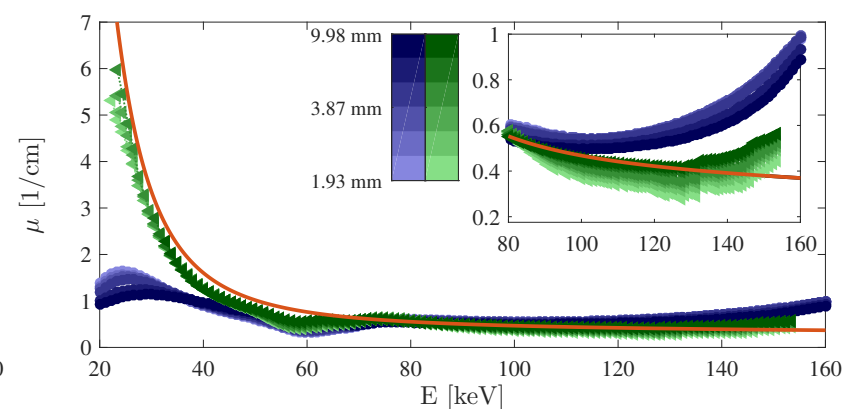

(b)

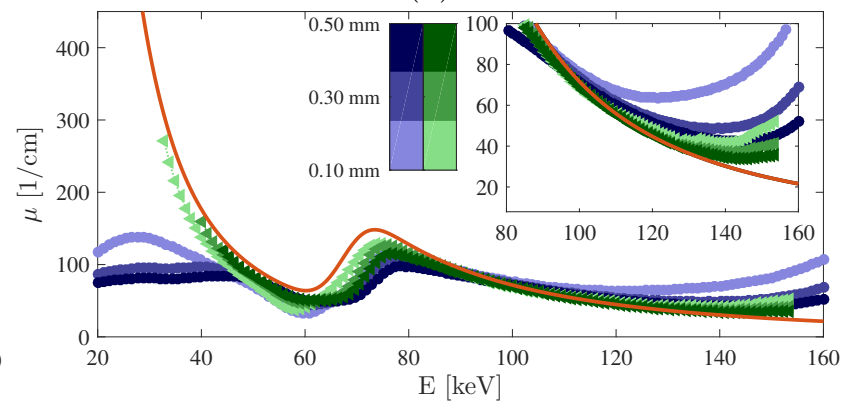

(d)

Figure 9. Comparison between raw (blue circles) and corrected (green triangles) attention curves of PVC (a), aluminium (b), copper (b), and Tantalum (d) at a flat field flux of $\Phi_{0}=4.7 \mathrm{Mph} / \mathrm{s} / \mathrm{mm}^{2}$. The color scales of corrected and raw attenuation curves show the thickness of the measured material. The theoretical curves (red) are convoluted with a Gaussian kernel with $\sigma=4 \mathrm{keV}$.

table, the corrected curves match the theory much better than the raw curves, but more importantly, it also match each other better. The later means that the correction algorithm seems to give a more consistent result of the linear attenuation coefficient between different material thicknesses compared to the raw data. This will make it possible to obtain a better material identification. Future work will investigate the efficiency of the correction algorithm in material identification applications.

\section{LIMITATION AND PERFORMANCE}

The algorithm presented in this paper is made to correct Multix ME100 v2 detector spectrum for different distorting effects. The approach is optimized for this detector, but the models are directly convertible to any CdTe line array detector, and could with small adjustments be used for 2D CdTe flat-panel detector or even CZT detectors. The algorithm uses semi-analytical interpretations of the different effects to do this. The models used are not full physical descriptions of the effects in the detector, but merely reasonable descriptions useful for correcting the spectra. Since our approach utilize the method of stripping, the individual corrections can easily be adjusted independently if better models are found for one of the effects. The algorithm works well up to fluxes of $5 \mathrm{Mph} / \mathrm{s} / \mathrm{mm}^{2}$. 


\subsection{Computation Time}

The correction algorithm is based on Matlab R2016a, and extensive optimization of computing time have not yet been perform. At present, the algorithm is capable of correcting the spectra of a full Multix ME100 detector (128 pixels) in $140 \pm 10 \mathrm{~ms}$ on a standard laptop. It is in particular the two iterative approaches SDC algorithm and the Pileup correction algorithm that add to the computation time. The SDC algorithm takes around $66 \%$ of the computational time whereas pileup correction algorithm takes $22 \%$. The remaining corrections only take $12 \%$ of the correction time. There is therefore a great potential to reduce computation time by optimizing the iterative parts of the correction algorithm. Further, every pixel is corrected individually meaning that the present algorithm can easily be parallelized, which, without any further optimization, would reduce the correction time to that of the individual pixel ( $\sim 10 \mathrm{~ms})$. It is therefore our estimate that slight optimization of the algorithm architecture can bring the correction time for the 128 spectra of an multix detector down close to the typical acquisition time of $1 \mathrm{~ms}$, making it usable in applications where material identification needs to be done fast, eg. luggage screening in airports.

\subsection{Setup Compatibility}

The pileup and SDC coefficients are together with the energy calibration, the only parameters that need to be adjusted for the individual Multix ME100 detectors and experimental set-ups. Therefore, the proposed correction algorithm can very easily be used in other experimental set-ups. The calibration of the algorithm only requires a measurement of the attenuation coefficient of a well defined material, like aluminium, at different thicknesses and at some different flat field fluxes. Furthermore, an energy calibration measurement might be needed. This can be done with a source with three well known peaks, including escape peaks. Such sources could be X-ray fluorescence from metals or a radioactive source where the peaks are well known.

\section{CONCLUSION}

We have in this paper shown an effective algorithm for correcting the spectrum of the high flux Multix ME100 v2 multi-spectral CdTe detector for effects like charge sharing, weighting potential cross talk, pulse pileup etc. Using synchrotron and laboratory sources, the different correction models have been verified and the algorithm has been tested. The results from calculating the linear attenuation curves from the corrected flat field and the attenuate spectra indicate that a better material identification is possible if the correction algorithm is applied to the raw data. The present algorithm is even with its iterative correction models, reasonably fast, correcting a 128 channel spectrum for the 128 pixel of one detector in $140 \mathrm{~ms}$, and it could easily be improved by one or two orders of magnitude, making it useful in application like luggage screening in airports. The algorithm is applicable in any set-up with the Multix ME100 v2 detector, requiring only a few calibration measurements using easily accessible materials, and could be extended to other pixelated CdTe and CZT detectors with small adjustments.

\section{ACKNOWLEDGMENTS}

The Authors would like to thank Exruptive for financing the ESRF beam time, through the initiative LINX (linking industry with neutrons and x-rays) funded by Innovations fund Denmark. Furthermore we thank Jon Wright from ID-11 for assistance during the beam time. and E.D. Christensen, Y. Gu, J. Kehres and U.L. Olsen acknowledges Innovation Fund Denmark for financing their respective work.

\section{REFERENCES}

[1] Beldjoudi, G., Rebuffel, V., Verger, L., Kaftandjian, V., and Rinkel, J., "Multidimensional data processing methods for material discrimination using an ideal X-ray spectrometric photon counting detector," IEEE Trans Nucl Sci 58(6) (2001).

[2] Martin, L., Enhanced information extraction in the multi-energy X-ray tomography for security, phd thesis, Boston University (2014).

[3] Yuan, Y., Tracey, B., and Miller, E., "Robust X-ray based material identification using multi-energy sinogram decomposition," Proc. SPIE 9847, Anomaly Detection and Imaging with X-rays 9847(98470V) (2016). 
[4] Kimoto, N., Hayashi, H., Asahara, T., Kanazawa, Y., Yamakawa, T., Yamamoto, S., Yamasaki, M., and Okada., M., "Development of a novel method based on a photon counting technique with the aim of precise material identification in clinical X-ray diagnosis," Proc. SPIE, Medical Imaging 2017 10132(1013239) (2017).

[5] Brambilla, A., Ouvrier-Buffet, P., Gonon, G., Rinkel, J., Moulin, V., Boudou, C., and Verger, L., "Fast CdTe and CdZnTe semiconductor detector arrays for spectroscopic X-ray imaging," IEEE Trans Nucl Sci 60(1) (2013).

[6] Gorecki, A., Brambilla, A., Moulin, V., Gaborieau, E., Radisson, P., and Verger, L., "Comparing performances of a CdTe X-ray spectroscopic detector and an X-ray dual-energy sandwich detector," J. Instrum. 8 (2013).

[7] Jimenez, E. S., Collins, N. M., Holswade, E. A., Devonshire, M. L., and Thompson., K. R., "Developing imaging capabilities of multi-channel detectors comparable to traditional X-ray detector technology for industrial and security applications," Proc SPIE 9969, Radiation Detectors: Systems and Applications 17(99690A) (2016).

[8] Cammin, J., Xu, J., Barber, W. C., Iwanczyk, J. S., Hartsough, N. E., and Taguchi, K., "A cascaded model of spectral distortions due to spectral response effects and pulse pileup effects in a photon-counting X-ray detector for CT," Med. Phys. 41(4) (2014).

[9] Fernandez, J. E., Scot, V., and Sabbatucci, L., "A modeling tool for detector resolution and incomplete charge collection," X-ray Spectrom. 44, 177-182 (2015).

[10] Cammin, J., Kappler, S., Weidinger, T., and Taguchi, K., "Evaluation of models of spectral distortions in photon-counting detectors for computed tomography," J. Med. Imaging 3(023503) (2016).

[11] Guerra, P., Santos, A., and Darambara, D. G., "Development of a simplified simulation model for performance characterization of a pixellated CdZnTe multimodality imaging system," Phys. Med. Biol. 53, 1099-1113 (2008).

[12] Plagnard, J., "Comparison of measured and calculated spectra emitted by the X-ray tube used at the Gustave Roussy radiobiological service," X-ray Spectrom. 43, 298-304 (2014).

[13] Castro, E. D., Pani, R., Pellegrini, R., and Bacci, C., "The use of cadmium telluride detectors for the qualitative analysis of diagnostic X-ray spectra," Phys. Med. Biol 29(9), 1117-1131 (1984).

[14] Knoll, G. F., [Radiation Detection and Measurement], John Wiley \& sons, Inc., 3 ed. (1999).

[15] Barrett, H. H., Eskin, J. D., and Barber, H. B., "Charge transport in arrays of semiconductor gamma-ray detectors," Phys. Rev. Lett. 75(1) (1995).

[16] Redus, R. H., Pantazis, J. A., Pantazis, T. J., Huber, A. C., and Cross, B. J., "Characterization of CdTe detectors for quantitative X-ray spectroscopy," IEEE Trans Nucl Sci 56, 2524-2532 (August 2009).

[17] Toyama, H., Higa, A., Yamazato, M., Maehama, T., R.Ohno, and Toguchi, M., "Quantitative analysis of polarization phenomena in CdTe radiation detectors," Jpn. J. Appl. Phys. 45(11) (2006).

[18] Thompson, A. and Vaughan, D., [X-ray Data Booklet], Lawrence Berkeley National Laboratory (2009).

[19] Bambynek, W., Crasemann, B., Fink, R. W., Freund, H. U., Mark, H., Swift, C. D., Price, R. E., and Rao, P. V., "X-ray fluorescence yields, Auger, and Coster-Kronig transition probabilities," Rev. Mod. Phys. 46(853) (1974).

[20] NIST, "X-ray form factor, attenuation, and scattering tables." http://physics.nist.gov/PhysRefData/FFast/ html/form.html (Retrieved 08-06-2017).

[21] Wang, X., Meier, D., Mikkelsen, S., Maehlum, G. E., Wagenaar, D. J., Tsui, B. M. W., Patt, B. E., and Frey, E. C., "Micro CT with energy-resolved photon-counting detectors," Phys Med Biol 56, 2791-2816 (Apri 2011).

[22] Hamel, L. A., Benoit, M., Donmez, B., Macri, J. R., McConnell, M. L., Narita, T., and Ryan, J. M., "Optimization of single-sided charge-sharing strip detectors," 2006 IEEE Nuclear Science Symposium Conference Record (2006).

[23] McGregor, D. S. and Hermon, H., "Room-temperature compound semiconductor radiation detectors," Nucl. Instrum. Methods Phys. Res., Sect. A 395(101) (1997).

[24] Iniewski, K., Chen, H., Bindley, G., Kuvvetli, I., and Budtz-J??rgensen, C., "Modeling charge-sharing effects in pixellated CZT detectors," 2007 IEEE Nuclear Science Symposium Conference Record (2007). 
[25] Shockley, W., "Currents to conductors induced by a moving point charge," J. Appl. Phys. 9(635) (1938).

[26] Ramo, S., "Currents induced by electron motion," Proc. IRE 27(9) (1939).

[27] Kehres, J., Lyksborg, M., and Olsen, U. L., "Threat detection of liquid explosives and precursors from their X-ray scattering pattern using energy dispersive detector technology," Submitted to SPIE Optics and Photonics (2017).

[28] NIST, "X-ray transition energies by element." http://physics.nist.gov/PhysRefData/XrayTrans/Html/ search.html (Retrieved 08-06-2017). 